\title{
Monte Carlo Simulation as an Auxiliary Tool for Electron Beam Quality Specification for Intra-Operative Radiotherapy
}

\author{
Aluísio José de Castro Neto, Cecília Maria Kalil Haddad, Edílson Lopes Pelosi, \\ Hospital Sírio Libanês, 01308-050, São Paulo, SP, Brazil
}

\author{
Juan Yury Zevallos-Chávez, Hélio Yoriyaz, and Paulo de Tarso Dalledone Siqueira \\ Instituto de Pesquisas Energéticas e Nucleares, Caixa Postal 11049, 05422-970, São Paulo, SP, Brazil
}

Received on 2 August, 2005

\begin{abstract}
Intra-operative radiotherapy (IORT) using electron beams has demonstrated to be a good alternative as part of the breast-conserving surgery. Besides, as the computer processing capacity has been increasing along the years, it has become a potential auxiliary tool in radiotherapy treatment planning. In this work these streams are merged together: simulations were performed by different Monte Carlo radiation transport codes (EGS4 and MCNP in its releases $4 \mathrm{C}$ and 5) in an attempt to not only examine the efficacy of $\mathrm{Al}$ and $\mathrm{Pb}$ discs used in IORT procedures to protect critical regions but also to compare the performance of the forementioned codes.
\end{abstract}

\section{INTRODUCTION}

Radiotherapeutic treatment success relies on delivering high doses to tumor cells while submitting the healthy tissue to as low as possible doses. Due to its low energy deposition range, electron beams are widely used in the treatment of superficial tumors but they are also suitable to intra-operative radiotherapy (IORT) $[1,2]$.

The IORT is a radiation technique whichs consist in delivering a high irradiation dose during the tumor removing surgery, right after the tumor removal. Besides taking advantage of the direct access of the radiation field to the irradiation site, IORT may also permit to preserve healthy neighbouring organs either by dislodging them from the bulk of the radiation field or by interposing shieldings between them and the target area. These IORT characteristics converge to attain better radiotherapeutic conditions than the traditional techniques

Preliminary researches on IORT given as a boost after breast-conserving surgery have demonstrated high capacity to decrease recurrence rate. However, the reliability of this procedure depends among several factors on the quality of previous dose measurements to determine the optimum condition for boost application and it becomes crucial specifically for electron beam . Typical electron beam energy ranges from 6 to $20 \mathrm{MeV}$ and the percentage depth doses (PDD) are measured through the utilization of ionization chambers. To help medical physicists to evaluate the dose values due to direct application of an electron boost we have simulated the process using Monte Carlo method to calculate energy deposited in water. In addition, the effects of the presence of an Al-Pb shielding disc in a breast irradiation configuration have also been analyzed.

As the computer processing capability has been increasing along the years, Monte Carlo methods have become a potential auxiliary tool for the determination of clinical parameters in radiotherapy treatment planning and particularly for the radiation beam characterization. EGS4 [3] and MCNP [4, 5] stand as 2 radiation transport codes which rely in Monte Carlo method. They have been used in this work to simulate an electron beam specification routine procedure. This proce- dure consists in measuring the depth dose profile in a water phantom which is stricken by an electron beam.

Results of the depth dose profile obtained by the two different Monte Carlo radiation transport codes, EGS4 and MCNP, due to electron beams impinging on a water phantom are presented. It is also presented the simulated results of depth dose profile obtained for a breast-IORT configuration with the shielding discs to evaluate their effect on preserving lungs from radiation exposition.

\section{SIMULATIONS}

Simulations were carried out by EGS4 and MCNP-4C as well as by its newest released version 5 and were divided in two sets. With the first set it is intended to provide simple experimental conditions to easily understand the differences on codes responses and to stress some difficulties on modelling. On the other hand, the second set shows the effects of a $\mathrm{Al}-\mathrm{Pb}$ shielding disc in breast-IORT configuration.

\section{A. Water Phantom}

The basic geometric configuration of the first set consists in a electron beam impinging on a cylindrical $(\mathrm{R}=7.5 \mathrm{~cm} ; \mathrm{H}=$ $12.0 \mathrm{~cm}$ ) water phantom. The monoenergetic electron source was made to emerge perpendicularly from a square sized (10 $\mathrm{cm} \times 10 \mathrm{~cm}$ ) surface placed $1.0 \mathrm{~cm}$ away from the top surface of the phantom. This phantom was immersed in an air medium. Depth dose profile was obtained by scoring the energy deposited in $3.60 \mathrm{~cm}^{3}(r=3.385 \mathrm{~cm}, h=0.1 \mathrm{~cm})$ cylindrical volumes centered along the simmetry axis.

Figure 1 shows the DDP for 5 distinct electron beam energies.

As it was expected, the electron Bragg peak drifts to the right (deeper profundities) as the beam energy increases, however other three main qualitatively points rise from the observation of this figure: 

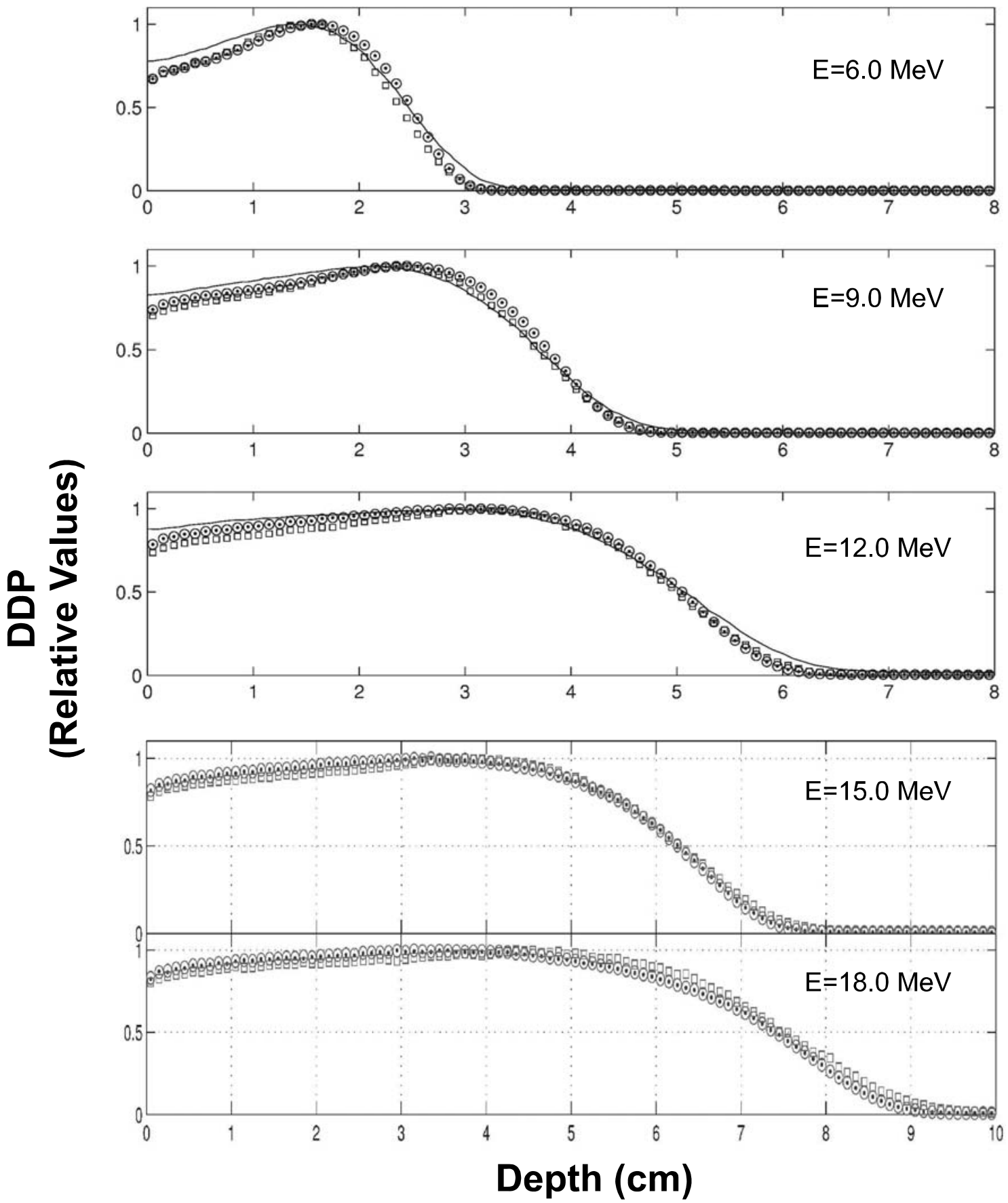

FIG. 1: Experimental Depth Dose Profile (solid line) and the simulated results obtained by EGS4 (squares), MCNP-4C (dots) and MCNP5 (circles) for five distinct electron beam energies: $\mathrm{E}=6.0 \mathrm{MeV}, \mathrm{E}=9.0 \mathrm{MeV}, \mathrm{E}=12.0 \mathrm{MeV}, \mathrm{E}=15.0 \mathrm{MeV}$ and $\mathrm{E}=18.0 \mathrm{MeV}$. Experimental values for the two higher energies are not still available.

1. MCNP-4C and MCNP5 give the same results which indicate there seems to be no new electron transport implement in the newest version;

2. As can be observed in figure 1, the electron Bragg peak obtained by EGS4 drifts faster to the right than do the ones from MCNP. This aspect can be regarded either by relying these codes on different calculation methodologies or by the use of different energy cross sections or even by both. Regarding their performance, both codes have their processing time enlarged by the increase of the beam energy. Table I shows the processing time for $6.0 \mathrm{MeV}$ and $15.0 \mathrm{MeV}$ electron beams. EGS4 was run in a Alpha Machine while MCNP-4C and MCNP5 were run in a $2.4 \mathrm{GHz}$ Pentium 4 CPU. Although the performed simulations have run 1.6 times faster for MCNP5 
than for MCNP-4C, it can not be stated that they employ different calculation procedures because they were not compiled by the same compiler. Besides scoring the amount of energy deposited along the central axis, MCNP also provides the uncertainty related to these values. It also allows to score simultaneously the energy deposited in a wide variety of volume cells. The forementioned tasks are done by the EGS4 in a different way, that is to say, the statistical uncertainties are evaluated later, therefore the program saves time while it is running, otherwise scoring the energy deposited for several volume cells at the same time would not be practical. Finally in the EGS4 code the cross section must be calculated every time a material is chosen, differently from MCNP where there are libraries for the cross sections already existing for the materials.

\begin{tabular}{c|c|c} 
& $6 \mathrm{MeV}$ & $15 \mathrm{MeV}$ \\
\hline EGS4 & 6.6 & 3.6 \\
MCNP-4C & 70.8 & 28.3 \\
MCNP5 & 116.4 & 46.2
\end{tabular}

TABLE I: EGS4 and MCNP's time processing rate (KPH - thousand of histories per hour) for $6.0 \mathrm{MeV}$ and $15.0 \mathrm{MeV}$ electron beam energies.

3. The DDP measurements have uncertainties less than $1 \%$, following the standards established. In spite of the DDP values calculated by the Monte Carlo codes have also good precision, uncertainties values of less than $0.2 \%$ were attained in both codes, there are still some characteristics of the experimental DDP data which the codes did not fit, such as the initial raise and the final slope. In fact the radiation field are far more complicated than the nominal energy displayed by the electron gun machine $[6,7]$. Despite this beam simplification, monoenergetic beams can still provide a good glance on radiation conditions due to their good qualitative agreement.

\section{B. Breast-IORT}

The basic geometric configuration of the second simulation set consists in a cylindrical simmetric configuration in which 3 cylinders differing on constitution (densities and material composition) and height are displaced one on another along the central axis. They are all $15 \mathrm{~cm}$ diameter cylinders and the one representing the soft tissue $(\mathrm{H}=4.65 \mathrm{~cm})$ stands between the air $(\mathrm{H}=1 \mathrm{~cm})$ and the lung $(\mathrm{H}=7 \mathrm{~cm})$ cylinders. A 5 $\mathrm{cm}$ diameter monoenergetic electron beam also centered with respect to this configuration is made to emerge from the top of the air cylinder and to flow downstream impinging on the soft tissue cylinder. This configuration intends to represent a breast-IORT and the simulated results obtained for the related DDP stand as references values on evaluating the radiotherapeutic gains obtained by interposing a $\mathrm{Al}-\mathrm{Pb}$ disc between the irradiation target area and the lung.

Figure 2 shows the geometric representation of this configuration in which an arrangement of Aluminum and Lead

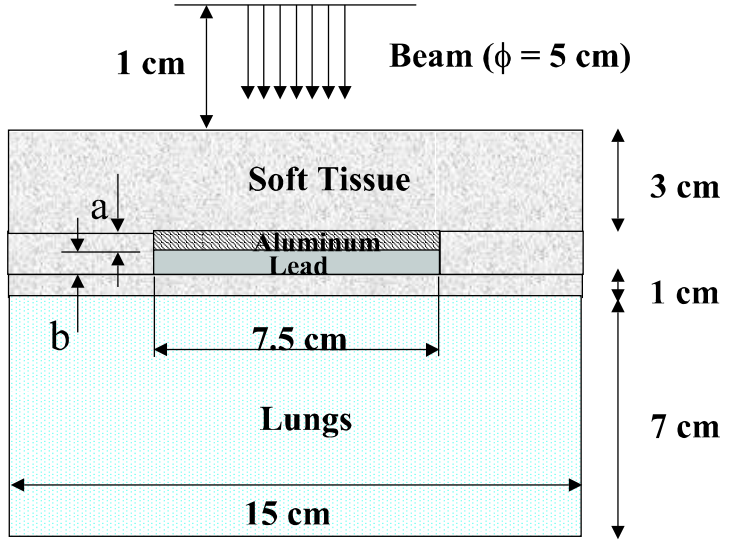

$a=0.25 \mathrm{~cm} \quad b=0.4 \mathrm{~cm}$

FIG. 2: Breast-IORT geometry with shielding discs.

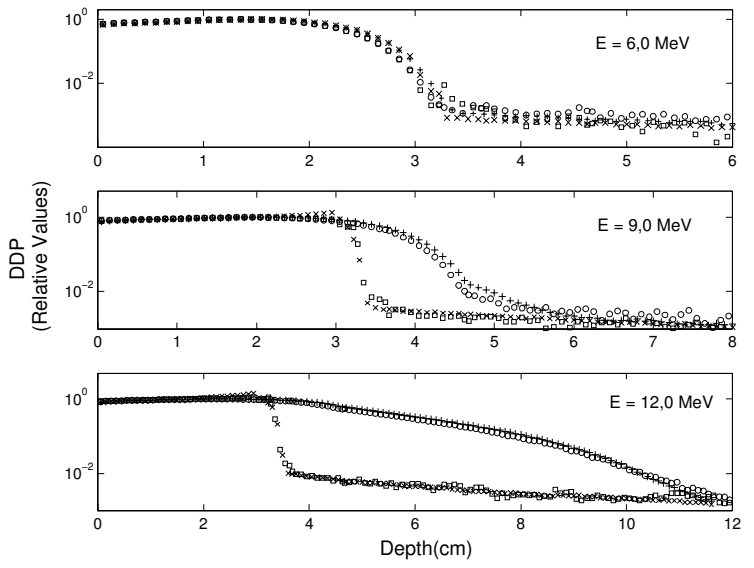

FIG. 3: Shielding discs effects evaluation in a breast-IORT. Depth Dose Profile obtained by EGS4 (circles: no shielding discs; squares: with shielding discs) and MCNP (plus: no shielding discs; cross : with shielding discs). MCNP stands for either MCNP versions.

shielding discs is introduced inside the soft tissue cylinder by simple constitution replacement.

This shielding arrangement is thought to fully stop the electron beam along the $\mathrm{Al}$ depth while the $\mathrm{Pb}$ disc would absorb the bremmstrahlung photons. A smaller electron reflection rate explains why $\mathrm{Al}$ is used to slowing down the electrons instead of $\mathrm{Pb}$. Figure 3 shows the changes on DDP due to the presence of the $\mathrm{Al}-\mathrm{Pb}$ disc for 3 distinc monoenergetic electron beams obtained by EGS and MCNP codes. In figure 4 is stressed the increasing effectiveness of the discs arrangement as a function of the electron beam energy, and the two peaks in this figure, observed for energies above $6 \mathrm{MeV}$, are due to the electron reflection in the discs. In order to show this results a normalization procedure was done, in which the normalization factor was the maximum DDP value obtained in the DDP 


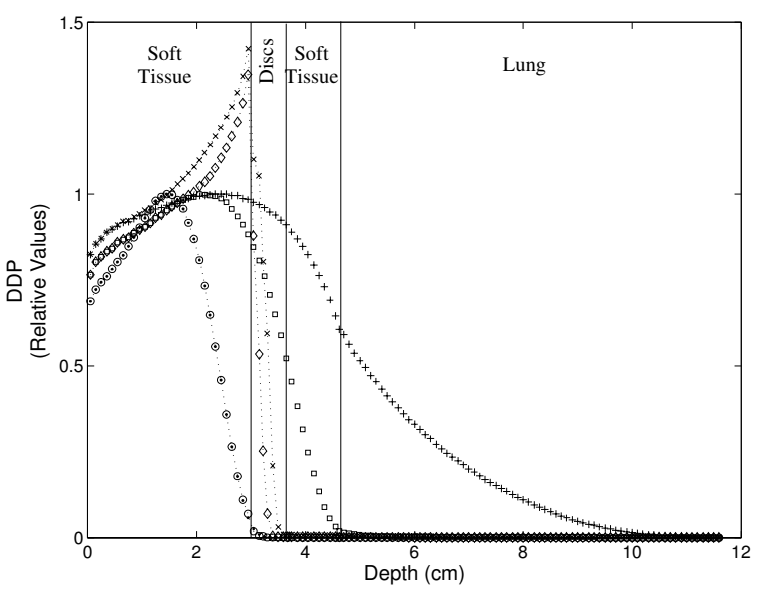

FIG. 4: Dependence of shielding discs efficiency on electron beam energy. (dots:- $6.0 \mathrm{MeV}$ without discs; circles: $6.0 \mathrm{MeV}$ with discs; squares: $9.0 \mathrm{MeV}$ without discs; diamonds: $9.0 \mathrm{MeV}$ with discs; plus: $12.0 \mathrm{MeV}$ without discs; cross : $12.0 \mathrm{MeV}$ with discs).

for $6 \mathrm{MeV}$ with no shielding discs arrangement.

As observed previously, the values obtained from these codes differ between each other, however they do present qua- litatively the same results. Shielding discs do play an important role on preventing higher doses to the lung for electron beam energies above $6 \mathrm{MeV}$, on the other hand backscattered electrons increase dose levels at irradiation target area. This change on dose distribution at irradiation target area demands an alteration on treatment planning.

\section{CONCLUSIONS}

Differences between the results obtained from these codes, may rely upon different calculations methodology and different physical models used by both Monte Carlo codes. However the influence of cross sections should be checked;

Time performance is not only influenced by CPU but also by the codes themselves and the used compiler;

EGS4 and MCNP are both suited to perform the simulations which can stand as important supporting device for electron beam quality specifications for radiotherapy, however time must be spent to a correct beam tuning;

The Al-Pb discs arrangement proposed in this work can reduce the dose imparted to lung in a IORT. The higher the electron beam energy the higher its efficiency on preventing undesirable doses, however the backscatter electrons disturbs the radiation field at the irradiation area.
[1] D. Bernard et al., Design optimization of intraoperative radiotherapy cones. Int. J. Radiation Oncology Biol. Phys. 55 (5) (2003).

[2] R. Orecchia et al., Intraoperative radiation therapy with electrons (ELIOT) in early-stage breast cancer. The Breast, 12 (2003).

[3] W. R. Nelson, D. W. O. Rogers, The EGS4 Code System. Standford Linear Accelerator, SLAC-265 (1984).

[4] J. D. Briesmeister, MCNP - A General Moste Carlo N-Particle Transport Code - Version 4C. (2000).
[5] J. D. Briesmeister, MCNP - A General Moste Carlo N-Particle Transport Code - Version 5. (2003).

[6] C. M. Ma, Characterizaion of computer simulated radiotherapy beams for Monte Carlo treatment planning. Radiat. Phys. Chem. 53 (1998).

[7] B. Faddegon et al., Clinical considerations of Monte Carlo for electron radiotherapy treatment planning. Radiat. Phy. Chem. 53 (1998). 